\title{
Comparative physiology of salt tolerance in Candida tropicalis and Saccharomyces cerevisiae
}

\author{
María J. García, Gabino Ríos, Rashid Ali,† Jose M. Bellés \\ and Ramón Serrano \\ Author for correspondence: Ramón Serrano. Tel: +3463877860 . Fax: +3463877859. \\ e-mail: serrano@ibmcp.upv.es
}

Instituto de Biologia

Molecular y Celular de

Plantas, Universidad

Politecnica de Valencia-CSIC,

Camino de Vera s/n, 46022

Valencia, Spain

\begin{abstract}
The salt tolerance of the respiratory yeast Candida tropicalis and the fermentative yeast Saccharomyces cerevisiae have been compared in glucose media. C. tropicalis showed a better adaptation to $\mathrm{Na}^{+}$and $\mathrm{Li}^{+}$and maintained higher intracellular $\mathrm{K}^{+}: \mathrm{Na}^{+}$and $\mathrm{K}^{+}: \mathrm{Li}^{+}$ratios than $\mathrm{S}$. cerevisiae. However, $\mathrm{C}$. tropicalis showed a poorer adaptation to osmotic stress (produced by $\mathrm{KCl}$ and sorbitol) and exhibited reduced glycerol production as compared to $S$. cerevisiae. In media with the non-repressing sugar galactose as carbon source, 5. cerevisiae exhibited reduced glycerol production and increased sensitivity to osmotic stress. Under these conditions, S. cerevisiae, but not C. tropicalis, utilized trehalose as a more important osmolyte than glycerol. These results suggest that the relative tolerance of yeast to the osmotic and cation toxicities of $\mathrm{NaCl}$, and the underlying relative capabilities for osmolyte synthesis and cation transport, are modulated by the general catabolite control exerted by glucose.
\end{abstract}

Keywords: salt stress, yeast, sodium, glycerol, trehalose

\section{INTRODUCTION}

The progressive salinization of irrigated land compromises the future of agriculture in the most productive areas of our planet (Ashraf, 1994). In this respect, a full understanding of the mechanisms of salt tolerance utilized by model organisms such as yeast could provide tools for the genetic engineering of salt-tolerant crops (Serrano \& Gaxiola, 1994). Micro-organisms, like many yeasts (Tokuoka, 1993), that can tolerate high saline environments, develop systems to counteract the deleterious effects of the two components of salt stress: intracellular ion toxicity and osmotic stress (turgor and water loss). In yeast, defence responses to salt stress are based on osmotic adjustment by osmolyte synthesis and cation transport systems for sodium exclusion. Polyols, and especially glycerol, are the major osmolytes produced by yeast (Blomberg \& Adler, 1992, 1993; Brown, 1990). Both a $\mathrm{Na}^{+}$-ATPase and a $\mathrm{H}^{+} / \mathrm{Na}^{+}$antiporter have been described as mechanisms of sodium extrusion (Serrano, 1996). Depending on the relative activities of

†Present address: National Institute for Biotechnology and Genetic Engineering, Jahng Road, Faisalabad, Pakistan. these systems, either the osmotic or the ionic toxicities of $\mathrm{NaCl}$ may dominate growth inhibition by salt stress.

The identification of the physiological factors which modulate the defence of yeast against salt stress is an important goal of salt tolerance studies. One important factor in yeast physiology is the type of sugar metabolism used, fermentative or respiratory (Gancedo \& Serrano, 1989). Glucose exerts a general modulation of metabolism and stress responses in yeast (Thevelein, 1994; Serrano, 1996). In addition, to meet with the energy requirements for metabolic and transport reactions (including stress-activated futile cycles; see Jennings, 1993), glucose metabolism has regulatory effects which are collectively described as either 'general catabolite control' (Gancedo \& Serrano, 1989) or 'glucose-induced signal transduction' (Thevelein, 1994). This phenomenon occurs in the so-called 'fermentative yeast', such as the genus Saccharomyces, where high rates of glucose catabolism down-regulate respiration (Gancedo \& Serrano, 1989) and defence responses against stresses such as heat shock, starvation (Thevelein, 1994; Serrano, 1996) and high salt concentrations (G. Rios \& R. Serrano, unpublished data). In the so-called 'respiratory yeast', such as the genus Candida, glucose catabolism is 
slow, the general catabolite control is not operative and most of the sugar is respired (Gancedo \& Serrano, 1989).

In this study we have compared the relative tolerance to osmotic stress and to alkali cation toxicity of the fermentative yeast Saccharomyces cerevisiae and the respiratory yeast Candida tropicalis. The results indicate that the operation of general catabolite control in $S$. cerevisiae, but not in C. tropicalis, correlates with a high capability for glycerol synthesis and osmotic tolerance but a low capability for sodium and lithium extrusion and cation tolerance. In addition, we have determined that, in the absence of glucose repression, trehalose behaves as an important osmoresponsive solute in $S$. cerevisiae.

\section{METHODS}

Yeast strains and growth. Candida tropicalis strain NCYC 2512 was isolated by R. Ali from a saline Pakistani soil on the basis of its tolerance to high salt concentrations $(15 \% \mathrm{NaCl})$. Saccharomyces cerevisiae strain DBY746 (MATahis3- $\Delta 1$ leu23,112 ura3-52 trp1-289) was obtained from the Yeast Genetic Stock Center, Berkeley, California. YPD medium containing $1 \%$ yeast extract (Difco), 2\% Bacto Peptone (Difco) and 2\% glucose was used for routine culture. Growth experiments were on SD minimal medium containing $2 \%$ glucose, $0.7 \%$ Yeast Nitrogen Base (without amino acids; Difco) and $50 \mathrm{mM}$ MES adjusted to $\mathrm{pH} 6.0$ with Tris. Histidine $\left(30 \mu \mathrm{g} \mathrm{ml}^{-1}\right)$, leucine $\left(100 \mu \mathrm{g} \mathrm{ml}^{-1}\right)$, uracil $\left(30 \mu \mathrm{g} \mathrm{ml}^{-1}\right)$ and tryptophan $\left(100 \mu \mathrm{g} \mathrm{ml}^{-1}\right)$ were also included in the case of $S$. cerevisiae. Saturated cultures of C. tropicalis and S. cerevisiae (grown for $3 \mathrm{~d}$ and with $\mathrm{OD}_{660}$ values of 5 and 2 , respectively) were diluted 50- and 30-fold, respectively, at time zero in normal medium or in medium containing $\mathrm{NaCl}(1 \mathrm{M}), \mathrm{KCl}(1 \mathrm{M})$, $\mathrm{LiCl}(0.2 \mathrm{M})$ or sorbitol $(1.5 \mathrm{M})$ as indicated. Growth was followed in $3 \mathrm{ml}$ cultures by $\mathrm{OD}_{660}$ using a Spectronic 20D (Milton Roy) spectrophotometer. The growth temperature was $28^{\circ} \mathrm{C}$ and the tubes were shaken by rotation on a tube roller (New Brunswick Scientific).

Intracellular cation determination. For $\mathrm{Li}^{+}$accumulation kinetics, $0 \cdot 1 \mathrm{M} \mathrm{LiCl}$ (final concentration) was added to a midexponential culture $\left(\mathrm{OD}_{660} 0.4-0.6 ; 500 \mathrm{ml}\right.$ in 21 shaken flask) and $20 \mathrm{ml}$ aliquots were collected at different times. Twenty millilitres of ice-cooled $180 \mathrm{mM}$ sorbitol and $20 \mathrm{mM} \mathrm{MgCl}_{2}$ were added and immediately centrifuged at 7000 r.p.m. in a Beckman JA- 20 rotor at $4{ }^{\circ} \mathrm{C}$. After resuspending in $10 \mathrm{ml}$ of the same ice-cooled sorbitol solution, cells were collected on glass microfibre filters (Whatman GF/C) and washed twice on the filter with the same solution. Extraction was performed in $1 \mathrm{ml} 20 \mathrm{mM} \mathrm{MgCl}_{2}$ at $95^{\circ} \mathrm{C}$ for $12 \mathrm{~min}$.

For $\mathrm{Na}^{+}$accumulation kinetics, $1 \mathrm{M} \mathrm{NaCl}$ (final concentration) was added as above and $20 \mathrm{ml}$ aliquots were collected at different times. Twenty millilitres of ice-cooled $1.5 \mathrm{M}$ sorbitol and $20 \mathrm{mM} \mathrm{MgCl}$, were added and cells were harvested by centrifugation at 7000 r.p.m. in a Beckman JA-20 rotor at $4{ }^{\circ} \mathrm{C}$. Pelleted cells were washed four times by centrifugation and resuspension in the same sorbitol solution. Extraction was performed as described above for lithium.

The ion content in the clarified extracts was determined with an Atomic Absorption Spectrometer (Varian) at flame emission mode. Internal water of C. tropicalis was determined by measuring the difference between total water of a cell pellet and extracellular water (Conway \& Armstrong, 1961). The latter was determined with $\mathrm{D}-\left[\mathrm{U}-{ }^{14} \mathrm{C}\right]$ sorbitol, a non-permeable and non-metabolizable compound for this yeast species (R. Ali, unpublished data). Determination of intracellular $\mathrm{K}^{+}$content was performed in the same extracts utilized for $\mathrm{Li}^{+}$and $\mathrm{Na}^{+}$determination. One $\mathrm{OD}_{660}$ unit of C. tropicalis suspension corresponded to an intracellular water content of $2 \cdot 4 \mu \mathrm{l}$ (ml suspension) $)^{-1}$. For $S$. cerevisiae one $\mathrm{OD}_{660}$ unit was equivalent to $1.6 \mu \mathrm{l}$ intracellular water (ml suspension) $)^{-1}$ (Gaxiola et al., 1992).

Glycerol determination. Samples $(1 \mathrm{ml})$ were withdrawn from mid-exponential cultures supplemented with $1 \mathrm{M} \mathrm{NaCl}$. Cells were collected by filtration through a glass microfibre filter (Whatman GF/C) and washed twice on the filter with a solution containing $1.5 \mathrm{M}$ sorbitol and $20 \mathrm{mM} \mathrm{MgCl}_{2}$. Cells were resuspended in $1 \mathrm{ml} \mathrm{0.5} \mathrm{M}$ Tris/ $\mathrm{HCl}, \mathrm{pH} \mathrm{7.6}$, and heated at $95{ }^{\circ} \mathrm{C}$ for $12 \mathrm{~min}$. After centrifugation, the supernatant was used to measure the internal glycerol content. For determination of extracellular glycerol, samples $(1 \mathrm{ml})$ were withdrawn from the culture, cells were removed by centrifugation and the clarified supernatant was used for the assay. Glycerol content was determined by means of an enzymic assay kit (Boehringer Mannheim, cat. no. 148270).

Trehalose determination. Extracts were prepared as described for glycerol determination except that water was used as the extracting medium. Separation of soluble carbohydrates was by high-performance anion-exchange chromatography coupled to pulsed amperometric detection (HPAE-PAD, Waters), using a CarboPac PA1 $(4 \times 250 \mathrm{~mm})$ column (Dionex) maintained at $22{ }^{\circ} \mathrm{C}$ (Rocklin \& Pohl, 1983).

\section{RESULTS AND DISCUSSION}

\section{Growth of C. tropicalis and S. cerevisiae in presence of different solutes}

Growth curves of C. tropicalis and S. cerevisiae in the absence and presence of $1 \mathrm{M} \mathrm{NaCl}$ are shown in Fig. 1 . C. tropicalis was able to grow much faster than $S$. cerevisiae due to its respiratory metabolism (Gancedo \&

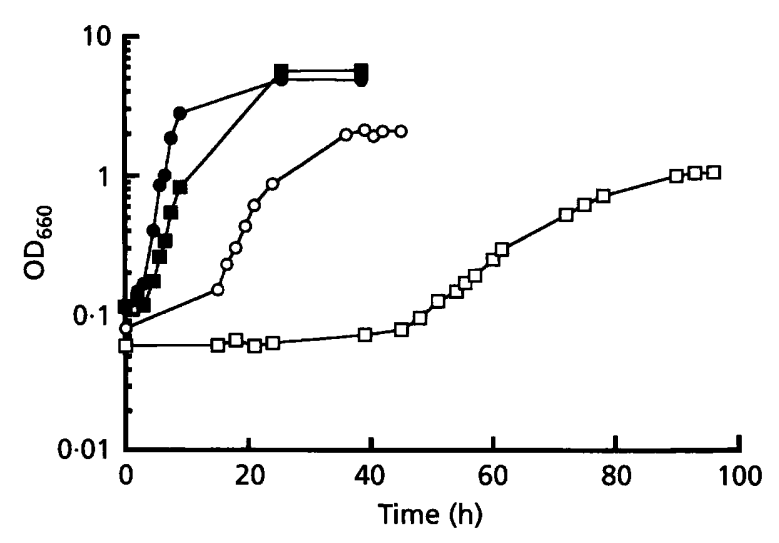

Fig. 1. Growth of $C$. tropicalis $(O, \square)$ and $S$. cerevisiae $(O, \square)$ in the absence $(0, O)$ and presence $(\square, \square)$ of $1 \mathrm{M} \mathrm{NaCl}$. Saturated cultures of $C$. tropicalis and $S$. cerevisiae $\left(\mathrm{OD}_{660}\right.$ values of 5 and 2, respectively) were diluted 50 - and 30 -fold, respectively, at time zero. Similar results were obtained in two independent experiments. 
Table 1. Relative effect of different solutes on growth of C. tropicalis and S. cerevisiae

Growth of C. tropicalis and S. cerevisiae was recorded after 14 and $26 \mathrm{~h}$, respectively. Results are expressed as a percentage of the $\mathrm{OD}_{660}$ value reached by control cultures in normal medium without additions. The absolute values were 3.5 for C. tropicalis and 1.5 for S. cerevisiae, corresponding to lateexponential phase (see Fig. 1). Values are the means of two determinations differing by less than $10 \%$.

\begin{tabular}{|lcc|}
\hline Solute & \multicolumn{2}{c|}{ Relative growth (\%) } \\
\cline { 2 - 3 } & C. tropicalis & S. cerevisiae \\
\hline None & 100 & 100 \\
$0.2 \mathrm{M} \mathrm{LiCl}$ & 10 & 2 \\
$1 \mathrm{M} \mathrm{NaCl}$ & 20 & 5 \\
$1 \mathrm{M} \mathrm{KCl}$ & 30 & 50 \\
$1.5 \mathrm{M} \mathrm{Sorbitol}$ & 3 & 30 \\
\hline
\end{tabular}

Serrano, 1989). In the absence of $\mathrm{NaCl}$ the growth rate of C. tropicalis was $0.43 \mathrm{~h}^{-1}$, whereas that of $S$. cerevisiae was $0.26 \mathrm{~h}^{-1}$. In the presence of $1 \mathrm{M} \mathrm{NaCl}, \mathrm{C}$. tropicalis showed a $30 \%$ reduction in growth rate while in the case of $S$. cerevisiae the reduction of growth rate was much greater, a $70 \%$ decrease. The lag phase was also dramatically increased (to $40 \mathrm{~h}$ ) in the case of $S$. cerevisiae in the presence of $1 \mathrm{M} \mathrm{NaCl}$. These results suggest that $C$. tropicalis grows better than $S$. cerevisiae at high $\mathrm{NaCl}$ concentrations, not only because of its more vigorous respiratory metabolism but also because of its higher salt tolerance.

We have investigated the sensitivities of both yeast species to different chloride salts $(\mathrm{NaCl}, \mathrm{KCl}, \mathrm{LiCl})$ and to sorbitol. Lithium is much more toxic than sodium while $\mathrm{KCl}$ is not toxic and only poses osmotic stress similar to sorbitol (Serrano, 1996). All these solutes produced an increase in both the generation time and the lag phase of yeast cultures (see Fig. 1 for $\mathrm{NaCl}$ ). We determined relative inhibitions by measuring growth at a fixed time-point, when control cultures had reached late exponential phase (Table 1 ). $\mathrm{KCl}$ was the least inhibitory solute for both $S$. cerevisiae and C. tropicalis. Taking $\mathrm{KCl}$ as a reference, sorbitol was strongly inhibitory for C. tropicalis but much better tolerated by S. cerevisiae. $\mathrm{NaCl}$ and $\mathrm{LiCl}$ were much better tolerated by $C$. tropicalis than by $S$. cerevisiae.

These results indicate that these yeast species differ in their relative sensitivities to osmotic stress (sorbitol) and cation toxicity (sodium and lithium). The low relative toxicity of $\mathrm{KCl}$ indicates that chloride poses no toxicity problem for either yeast species. The fact that $\mathrm{KCl}$ is much less inhibitory for C. tropicalis than sorbitol could be explained if $\mathrm{K}^{+}$uptake, but not sorbitol uptake, could be utilized for osmotic adjustment in this species (see below). The difference between $\mathrm{KCl}$ and sorbitol toxicities is much less apparent in $S$. cerevisiae.

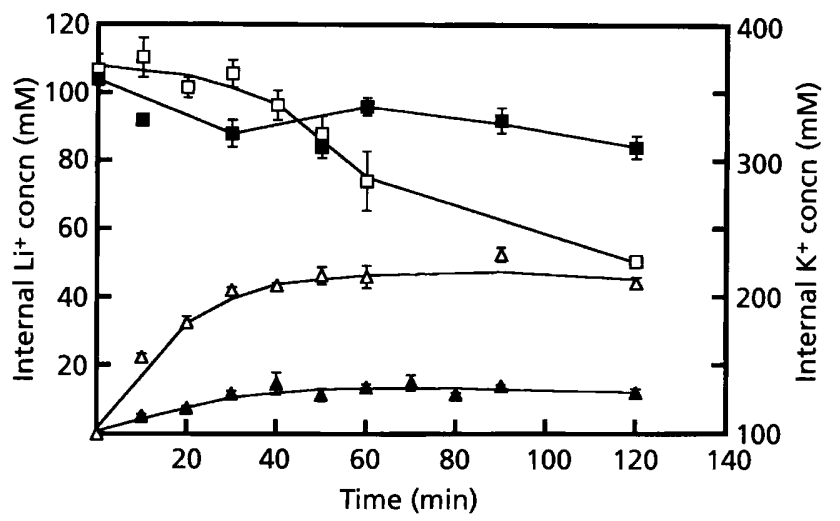

Fig. 2. Kinetics of $\mathrm{Li}^{+}$uptake $(\triangle, \boldsymbol{\Delta})$ and $\mathrm{K}^{+}$efflux $(\square, \boldsymbol{\square})$ in $C$. tropicalis $(\boldsymbol{\Lambda}, \square)$ and $S$. cerevisiae $(\triangle, \square)$. Cell cultures growing exponentially $\left(O D_{660} 0.4-0.6\right)$ were supplemented at time zero with $100 \mathrm{mM} \mathrm{LiCl}$ and samples were taken at the indicated times for intracellular cation determination as described in Methods. Values are the means of three independent determinations and bars represent the SD.

\section{Cation uptake and homeostasis}

The greater sensitivity of $S$. cerevisiae to $\mathrm{Na}^{+}$and $\mathrm{Li}^{+}$as compared to $C$. tropicalis suggested that these toxic cations could be accumulated to higher levels in the former yeast species. As indicated in Fig. $2\left(\mathrm{Li}^{+}\right.$ accumulation) and Fig. $3\left(\mathrm{Na}^{+}\right.$accumulation) this is actually the case. C. tropicalis accumulated much less $\mathrm{Li}^{+}$than $S$. cerevisiae, the differences being apparent from the earliest time point at only $10 \mathrm{~min}$ (Fig. 2). Therefore the cation uptake system must have different relative activities for $\mathrm{Li}^{+}$and $\mathrm{K}^{+}$(present in the medium) in the two yeast species. $\mathrm{Li}^{+}$accumulation caused no significant changes in intracellular $\mathrm{K}^{+}$levels during the first hour. At longer times the $\mathrm{K}^{+}$content of $S$. cerevisiae cells, but not of C. tropicalis cells, started to decrease. This could be a secondary effect of metabolic poisoning by high levels of intracellular $\mathrm{Li}^{+}$.

Initial $\mathrm{Na}^{+}$influx during the first $30 \mathrm{~min}$ was very similar in both yeast species (Fig. 3). However, at longer times C. tropicalis was able to maintain a lower intracellular steady-state $\mathrm{Na}^{+}$level than $S$. cerevisiae. According to the kinetic model developed for net accumulation of $\mathrm{Li}^{+}$and $\mathrm{Na}^{+}$in yeast (RodriguezNavarro \& Asensio, 1977; Rodriguez-Navarro \& Ortega, 1982), this suggests that the major differences in sodium transport systems between the two yeast species are concerned with active efflux of the cation. Both $C$. tropicalis and $S$. cerevisiae suffered a massive (almost $50 \%$ ) and rapid (completed in less than $10 \mathrm{~min}$ ) loss of internal $\mathrm{K}^{+}$after addition of $1 \mathrm{M} \mathrm{NaCl}$ (Fig. 3). This $\mathrm{K}^{+}$ efflux was not observed in the case of $0.1 \mathrm{M} \mathrm{LiCl}$ (Fig. 2) and it may be due to osmotic shock. Gustin et al. (1988) reported the presence of putative mechanosensitive channels in $S$. cerevisiae opened by the shrinkage produced by osmotic stress. These channels have a higher selectivity for $\mathrm{K}^{+}$than for other ions such as $\mathrm{Na}^{+}$. 


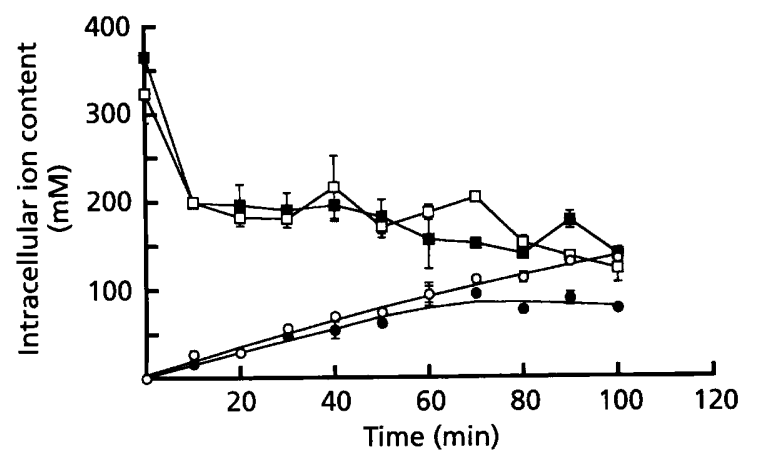

Fig. 3. Kinetics of $\mathrm{Na}^{+}$uptake $(O, 0)$ and $\mathrm{K}^{+}$efflux $(\square, \square)$ in $C$. tropicalis $(0, \square)$ and $S$. cerevisiae $(O, \square)$. Cell cultures growing exponentially $\left(O D_{660} 0.4-0.6\right)$ were supplemented at time zero with $1 \mathrm{M} \mathrm{NaCl}$ and samples were taken at the indicated times for intracellular cation determination as described in Methods. Values are the means of three independent determinations and bars represent the SD.

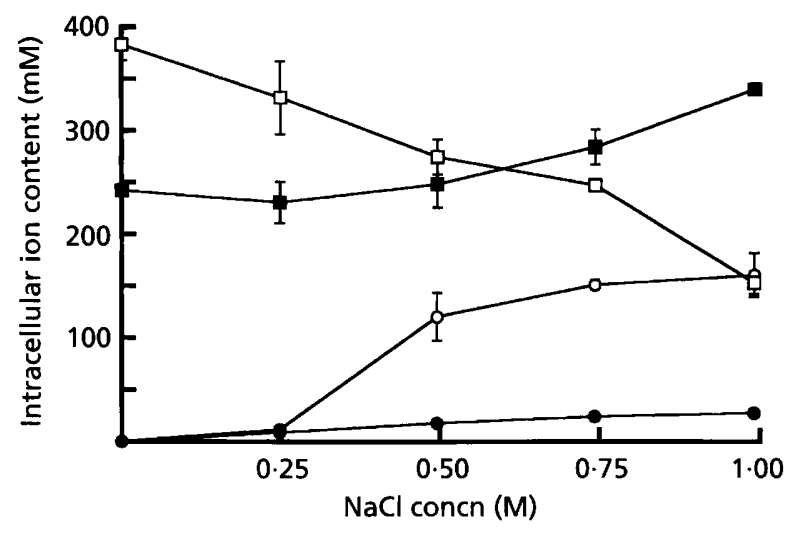

Fig. 4. Intracellular steady-state levels of $\mathrm{Na}^{+}(\mathrm{O}, 0)$ and $\mathrm{K}^{+}$ $(\square, \square)$ in C. tropicalis $(0, \square)$ and S. cerevisiae $(O, \square)$. Samples of cell cultures growing exponentially $\left(O D_{660} 0.4-0.6\right)$ with the indicated $\mathrm{NaCl}$ concentrations were taken for intracellular cation determination as described in Methods. Values are the means of three independent determinations and bars represent the SD.

It is known that intracellular $\mathrm{Na}^{+}: \mathrm{K}^{+}$ratios above $0 \cdot 5$ are toxic to yeast cells (Camacho et al., 1981; Gaxiola et al., 1992). For S. cerevisiae this ratio increased up to a value of $1 \cdot 1$ at the end of the experiment shown in Fig. 3. On the other hand, C. tropicalis maintained a $\mathrm{Na}^{+}: \mathrm{K}^{+}$ ratio lower than 0.5 during the whole uptake experiment.

The results of these kinetic experiments were corroborated by studies of the steady-state levels of $\mathrm{Na}^{+}$and $\mathrm{K}^{+}$during growth. For external $\mathrm{NaCl}$ concentrations of $\geqslant 0.5 \mathrm{M}$, S. cerevisiae accumulated higher amounts of $\mathrm{Na}^{+}$than C. tropicalis (Fig. 4). $\mathrm{K}^{+}$contents decreased at high $\mathrm{NaCl}$ concentrations in $S$. cerevisiae but increased in C. tropicalis. Therefore, after the initial $\mathrm{K}^{+}$loss triggered by $\mathrm{NaCl}$ addition in both yeast species (Fig. 3), there must be an adaptative response in C. tropicalis to recover the intracellular $\mathrm{K}^{+}$level and even to increase it at high $\mathrm{NaCl}$ concentrations. In $S$. cerevisiae $\mathrm{Na}^{+}: \mathrm{K}^{+}$ ratios were increased up to a value of about 1 in presence of $1 \mathrm{M} \mathrm{NaCl}$. However, in C. tropicalis the decrease in $\mathrm{Na}^{+}$uptake and the increase in $\mathrm{K}^{+}$retention resulted in low $\mathrm{Na}^{+}: \mathrm{K}^{+}$ratios even at high $\mathrm{NaCl}$ concentrations $\langle 0 \cdot 1$ at $1 \mathrm{M} \mathrm{NaCl})$. Moreover, C. tropicalis was able to grow at a $\mathrm{NaCl}$ concentration of $1.5 \mathrm{M}$, whereas $S$. cerevisiae could not. Under this condition, the intracellular $\mathrm{Na}^{+}: \mathrm{K}^{+}$ratio was only $0 \cdot 2$ (M. J. García, unpublished data).

In $S$. cerevisiae the intracellular $\mathrm{Na}^{+}$level obtained in kinetic experiments with $1 \mathrm{M} \mathrm{NaCl}(0 \cdot 15 \mathrm{M}$; Fig. 3) was very similar to the steady-state level achieved during growth at the same $\mathrm{NaCl}$ concentration (Fig. 4). In C. tropicalis, however, the steady-state level after 1-1.5 h of incubation with $1 \mathrm{M} \mathrm{NaCl}(0.09 \mathrm{M}$; Fig. 3) was much higher than the steady-state level during growth at this $\mathrm{NaCl}$ concentration ( $0.03 \mathrm{M}$; Fig. 4). This suggests the operation in C. tropicalis of an adaptative mechanism to increase sodium efflux or restrict sodium uptake during continuous growth in media with $\mathrm{NaCl}$.

Taken together, these results are evidence of a higher adaptation of C. tropicalis to ionic stress as compared to $S$. cerevisiae. It is known that $\mathrm{Na}^{+}$and $\mathrm{Li}^{+}$ions enter $S$. cerevisiae cells through the $\mathrm{K}^{+}$transporter (Gaber, 1992; Serrano, 1996). Cation uptake is more efficient at producing salt tolerance in C. tropicalis as compared to $S$. cerevisiae because (a) the initial rate of $\mathrm{Li}^{+}$uptake is decreased (Fig. 2) and (b) $\mathrm{K}^{+}$accumulates to higher levels in response to $\mathrm{NaCl}$ stress (Fig. 4) despite similar efflux (Fig. 3). In S. cerevisiae cation uptake is mediated by the TRK1,2 system (Gaber, 1992; Serrano, 1996). This system discriminates against $\mathrm{Na}^{+}$and $\mathrm{Li}^{+}$in favour of $\mathrm{K}^{+}$and this discrimination increases during salt stress (Haro et al., 1993). If the same system were operative in C. tropicalis, it should have an even greater discriminatory power and, in addition, should respond to salt stress by increased $\mathrm{K}^{+}$accumulation. A different $\mathrm{K}^{+}$ transporter encoded by the HAK1 gene has recently been identified in the yeast Schwanniomyces occidentalis (Bañuelos et al., 1995). This system is more efficient than the $S$. cerevisiae TRK1,2 system and therefore the presence of HAK1-related transporters in C. tropicalis should be investigated.

As discussed above, sodium efflux in C. tropicalis is more efficient than in S. cerevisiae. Sodium efflux in $S$. cerevisiae is mediated by the ENA1 ATPase (Haro et al., 1991), which is induced upon salt stress (Garciadeblas $e t$ al., 1993). C. tropicalis could have a similar ATPase but with higher activity and better up-regulation by salt stress. In S. cerevisiae, expression of the ENA1 gene is controlled by multiple signal transduction pathways (Marquez \& Serrano, 1996) which could be more active in C. tropicalis. Alternatively, a $\mathrm{H}^{+} / \mathrm{Na}^{+}$antiporter has been described in Schizosaccharomyces pombe (Jia et al., 1992) and Zygosaccharomyces rouxii (Watanabe et $a l ., 1995)$ and a high activity of this kind of transporter 


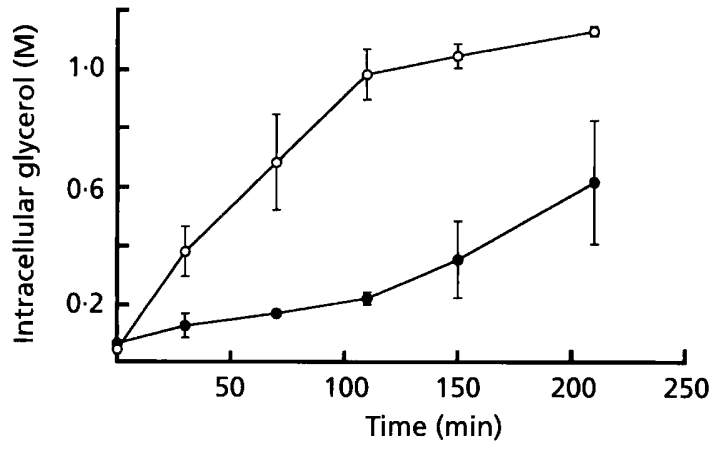

Fig. 5. Kinetics of intracellular glycerol accumulation in $C$. tropicalis $(\mathbf{O})$ and $S$. cerevisiae $(O)$ upon salt stress. Cell cultures growing exponentially in glucose medium $\left(O D_{660} 0.4-0.6\right)$ were supplemented at time zero with $1 \mathrm{M} \mathrm{NaCl}$ and samples taken at the indicated times for intracellular glycerol determination as described in Methods. Values are the means of three independent determinations and bars represent the SD.

Table 2. Intracellular concentrations of glycerol and trehalose in S. cerevisiae and C. tropicalis

Cells were grown to exponential phase $\left(\mathrm{OD}_{660} 0.4-0 \cdot 6\right)$ in media with either glucose or galactose as carbon source and with $1 \mathrm{M} \mathrm{NaCl}$ when indicated. Samples were taken for glycerol and trehalose determination as described in Methods. Values are the means of two determinations differing by less than $10 \%$. ND, not determined.

\begin{tabular}{|c|c|c|c|c|}
\hline \multirow[t]{3}{*}{ Growth medium } & \multicolumn{4}{|c|}{ Intracellular concentration $(\mathbf{M})$} \\
\hline & \multicolumn{2}{|c|}{ S. cerevisiae } & \multicolumn{2}{|c|}{ C. tropicalis } \\
\hline & Glycerol & Trehalose & Glycerol & Trehalose \\
\hline Glucose & $0 \cdot 04$ & $<0.02$ & $0 \cdot 06$ & $<0.02$ \\
\hline Glucose $+\mathrm{NaCl}$ & $1 \cdot 2$ & 0.05 & $1 \cdot 3$ & $<0.02$ \\
\hline Galactose & $<0.02$ & $0 \cdot 11$ & ND & $<0.02$ \\
\hline Galactose $+\mathrm{NaCl}$ & $0 \cdot 48$ & 0.75 & ND & $<0.02$ \\
\hline
\end{tabular}

could also explain the capability of C. tropicalis for sodium efflux.

\section{Glycerol production under salt stress}

In the case of $S$. cerevisiae growing on glucose, $\mathrm{Na}^{+}$ toxicity is the major problem associated with salinity and this can be explained by the reduced capability for sodium exclusion and potassium accumulation of $S$. cerevisiae compared with C. tropicalis. In the latter yeast species, however, the osmotic component of salt stress poses the greater problem during growth on $\mathrm{NaCl}$. This could be explained by a reduced capability of $C$. tropicalis for osmotic adjustment. As shown in Fig. 5, S. cerevisiae started to accumulate glycerol immediately after salt shock and reached steady-state levels in $2 \mathrm{~h}$. In C. tropicalis glycerol production was much slower and did not reach a steady-state level during the time course of the experiment. However, during steady-state growth with $1 \mathrm{M} \mathrm{NaCl} \mathrm{C}$. tropicalis was able to accumulate glycerol up to a concentration of $1.3 \mathrm{M}$, which is similar to that reached by $S$. cerevisiae (Table 2). This suggests that osmotic adjustment in glucose medium is achieved in both yeast species by glycerol accumulation, although in C. tropicalis glycerol synthesis is much slower than in S. cerevisiae. External glycerol concentration (less than $2 \mathrm{mM}$ ) did not change during the whole experiment in either of the strains tested (data not shown).

Studies carried out on over 20 different yeasts and filamentous fungi show that glycerol seems to be the main osmolyte accumulated upon salt stress (Blomberg \& Adler, 1992, 1993). Glycerol production involves $\mathrm{NADH}$-dependent reduction of dihydroxyacetone phosphate to glycerol 3-phosphate by cytoplasmic glycerol3-phosphate dehydrogenase (GDP) and subsequent dephosphorylation of glycerol 3-phosphate to glycerol. Growth in the presence of $\mathrm{NaCl}$ increased GPD activity about sevenfold (André et al., 1991; Blomberg \& Adler, 1989; Albertyn et al., 1994a) and a great part of this regulation is at the level of transcription (Albertyn et al., 1994b). It seems that high rates of glycolysis, as occur during catabolic repression by glucose in S. cerevisiae, are optimal for glycerol production. In C. tropicalis the rate of glycolysis is lower, no catabolic repression occurs and respiration occurs at high rates. Slow glycolysis will reduce the supply of dihydroxyacetone phosphate and the high respiration rate will consume both dihydroxyacetone phosphate and NADH, thus competing with their utilization for glycerol synthesis (Gancedo \& Serrano, 1989). Although GPD is repressed by glucose in $S$. cerevisiae, salt induction overcomes the repression effect and the enzyme has high activity in media with glucose and $\mathrm{NaCl}$ (Albertyn et al., 1994a).

We have recently observed that $S$. cerevisiae growing in galactose media has high osmotic sensitivity because sorbitol and $\mathrm{KCl}$ are as toxic as $\mathrm{NaCl}$ at equivalent osmotic concentrations. In addition, glycerol synthesis is greatly reduced under these conditions (G. Ríos \& R. Serrano, unpublished data). Galactose is metabolized more slowly than glucose and is not as repressive as glucose. Therefore, the energy metabolism of $S$. cerevisiae on galactose medium is mostly respiratory (Gancedo \& Serrano, 1989).

One unexpected finding indicated in Table 2 is that trehalose is a better osmolyte than glycerol for $S$. cerevisiae growing on galactose medium with $\mathrm{NaCl}$. Trehalose is not accumulated in C. tropicalis under any conditions. Trehalose is not considered as an osmoregulatory osmolyte in $S$. cerevisiae, mostly because it is not accumulated during osmotic stress on glucose medium (Brown et al., 1986). Trehalose accumulation has only been observed upon heat shock (Hottiger $e t$ al., 1987) and starvation during sporulation or stationary phase (Gancedo \& Serrano, 1989). Our results indicate that, when the repressing effect of glucose is removed, as in galactose medium, trehalose is an osmoregulatory solute contributing more to the osmotic adjustment than glycerol (Table 2). Trehalose phosphate synthase 
(Gancedo \& Serrano, 1989) and trehalose phosphate phosphatase (Gounalaki \& Thireos, 1994) are repressed by glucose while trehalase is activated by glucose (Gancedo \& Serrano, 1989). Therefore, glucose should strongly oppose trehalose synthesis, even in the presence of stresses such as $\mathrm{NaCl}$ which induce trehalose synthesis. Only in non-repressing carbon sources such as galactose can the osmoregulatory role of trehalose be observed. We have observed that a $S$. cerevisiae tps1 mutant, devoid of trehalose-6-phosphate synthase and unable to grow on glucose medium (Thevelein, 1994), is more sensitive to osmotic stress produced by sorbitol or $\mathrm{KCl}$ on galactose medium than wild-type $(\mathrm{G}$. Rios \& $\mathrm{R}$. Serrano, unpublished observations). This corroborates the suggested role of trehalose as an osmoresponsive compatible solute on galactose medium.

As indicated in Table 2, trehalose is not accumulated in C. tropicalis despite the lack of catabolic repression of this yeast (Gancedo \& Serrano, 1989). It has been suggested that trehalose biosynthesis in $S$. cerevisiae plays a crucial role in modulating glycolysis (Thevelein, 1994). Apparently, the high rate of glucose phosphorylation of $S$. cerevisiae requires a pathway for consuming sugar phosphates which is provided by trehalose biosynthesis. This hypothesis is in accordance with the lack of trehalose biosynthesis in a yeast with slow glucose phosphorylation and glycolysis such as $C$. tropicalis.

\section{ACKNOWLEDGEMENTS}

This work was supported by a grant of the Project of Technological Priority of the European Commission (Brussels) to R. Serrano. R. Ali was supported by a fellowship of the International Atomic Energy Agency (Vienna, Austria). G. Rios is a fellow of the Spanish Ministerio de Educacion y Ciencia (Madrid). We thank Dr Avelino Corma and Maria Jesus Lacruz (Instituto de Tecnologia Quimica, Valencia, Spain) for making available their atomic absorption spectrophotometer and for assistance with cation measurements.

\section{REFERENCES}

Albertyn, J., Hohmann, S. \& Prior, B. A. (1994a). Characterization of the osmotic-stress response in Saccharomyces cerevisiae: osmotic stress and glucose repression regulate glycerol-3-phosphate dehydrogenase independently. Curr Genet 25, 12-18.

Albertyn, J., Hohmann, S., Thevelein, J. M. \& Prior, B. A. (1994b). GPD1, which encodes glycerol-3-phosphate dehydrogenase, is essential for growth under osmotic stress in Saccharomyces cerevisiae, and its expression is regulated by the high-osmolarity glycerol response pathway. Mol Cell Biol 14, 4135-4144.

André, L., Hemming, A. \& Adler, L. (1991). Osmoregulation in Saccharomyces cerevisiae. Studies on the osmotic induction of glycerol production and glycerol 3-phosphate dehydrogenase $\left(\mathrm{NAD}^{+}\right)$. FEBS Lett 286, 13-17.

Ashraf, M. (1994). Breeding for salinity tolerance in plants. Crit Rev Plant Sci 13, 17-42.

Bañuelos, M. A., Klein, R. D., Alexander-Bowman, S. J. \& Rodriguez-Navarro, A. (1995). A potassium transporter of the yeast Schwanniomyces occidentalis homologous to the Kup system of Escherichia coli has a high concentrative capacity. EMBO J 14, 3021-3027.

Blomberg, A. \& Adler, L. (1989). Roles of glycerol and glycerol-3phosphate dehydrogenase $\left(\mathrm{NAD}^{+}\right)$in acquired osmotolerance of Saccharomyces cerevisiae. J Bacteriol 171, 1087-1092.

Blomberg, A. \& Adler, L. (1992). Physiology of osmotolerance in fungi. Adv Microb Physiol 33, 145-212.

Blomberg, A. \& Adler, L. (1993). Tolerance of fungi to $\mathrm{NaCl}$. In Stress Tolerance of Fungi, pp. 209-232. Edited by D. H. Jennings. New York: Marcel Dekker.

Brown, A. D. (1990). Microbial Water Stress Physiology. New York: John Wiley \& Sons.

Brown, A. D., Mackenzie, K. F. \& Singh, K. K. (1986). Selected spects of microbial osmoregulation. FEMS Microbiol Rev 39, 31-36.

Camacho, M., Ramos, J. \& Rodriguez-Navarro, A. (1981). Potassium requirements of Saccharomyces cerevisiae. Curr Microbiol 6, 295-299.

Conway, E. J. \& Armstrong, W. MCD. (1961). The total intracellular concentration of solutes in yeast and other plant cells and the distensibility of the plant-cell wall. Biochem J 81, 631-639.

Gaber, R. F. (1992). Molecular genetics of yeast ion transport. Int Rev Cytol 137A, 299-353.

Gancedo, C. \& Serrano, R. (1989). Energy-yielding metabolism. In The Yeasts, 2nd edn, vol. 3, pp. 205-259. Edited by J. S. Harrison $\&$ A. H. Rose. New York: Academic Press.

Garciadeblas, B., Rubio, F., Quintero, F. J., Bañuelos, M. A., Haro, R. \& Rodriguez-Navarro, A. (1993). Differential expression of two genes encoding isoforms of the ATPase involved in sodium efflux in Saccharomyces cerevisiae. Mol Gen Genet 236, 363-368.

Gaxiola, R., de Larrinoa, I. F., Villalba, J. M. \& Serrano, R. (1992). A novel and conserved salt-induced protein is an important determinant of salt tolerance in yeast. EMBO J 11, 3157-3164.

Gounalaki, N. \& Thireos, G. (1994). Yap1p, a yeast transcriptional activator that mediates multidrug resistance, regulates the metabolic stress response. EMBO J 13, 4036-4041.

Gustin, M. C., Zhou, X.-L., Martinac, B. \& Kung, C. (1988). A mechanosensitive ion channel in the yeast plasma membrane. Science 242, 762-765.

Haro, R., Garciadeblas, B. \& Rodriguez-Navarro, A. (1991). A novel P-type ATPase from yeast involved in sodium transport. FEBS Lett 291, 189-191.

Haro, R., Bañuelos, M. A., Quintero, F. J., Rubio, F. \& RodriguezNavarro, A. (1993). Genetic basis of sodium exclusion and sodium tolerance in yeast. A model for plants. Plant Physiol 89, 868-874.

Hottiger, T., Schmutz, P. \& Wiemken, A. (1987). Heat-induced accumulation and futile cycling of trehalose in Saccharomyces cerevisiae. J Bacteriol 169, 5518-5522.

Jennings, D. H. (1993). Understanding tolerance to stress: laboratory culture versus environmental actuality. In Stress Tolerance of Fungi, pp. 1-12. Edited by D. H. Jennings. New York: Marcel Dekker.

Jia, Z.-P., McCullough, N., Martel, R., Hemmingsen, S. \& Young, P. G. (1992). Gene amplification at a locus encoding a putative $\mathrm{Na}^{+} / \mathrm{H}^{+}$antiporter confers sodium and lithium tolerance in fission yeast. $E M B O J 11,1631-1640$.

Marquez, J. A. \& Serrano, R. (1996). Multiple transduction pathways regulate the sodium-extrusion gene PMR2/ENA1 during salt stress in yeast. FEBS Lett 382, 89-92.

Rocklin, R. D. \& Pohl, C. A. (1983). Determination of carbo- 
hydrates by anion exchange chromatography with pulsed amperometric detection. J Liquid Chromatogr 6, 1577-1590.

Rodriguez-Navarro, A. \& Asensio, J. (1977). An efflux mechanism determines the low net entry of lithium in yeast. FEBS Lett 75, 169-172.

Rodriguez-Navarro, A. \& Ortega, M. D. (1982). The mechanism of sodium efflux in yeast. FEBS Lett 138, 205-208.

Serrano, R. (1996). Salt tolerance in plants and microorganisms: toxicity targets and defense reponses. Int Rev Cytol 165, 1-52.

Serrano, R. \& Gaxiola, R. (1994). Microbial models and salt stress tolerance in plants. Crit Rev Plant Sci 13, 121-138.
Thevelein, J. M. (1994). Signal transduction in yeast. Yeast 10 , 1753-1790.

Tokuoka, K. (1993). Sugar- and salt-tolerant yeast. J Appl Bacteriol 74, 101-110.

Watanabe, Y., Miwa, S. \& Tamai, Y. (1995). Characterization of $\mathrm{Na}^{+} / \mathrm{H}^{+}$-antiporter gene closely related to the salt-tolerance of yeast Zygosaccharomyces rouxii. Yeast 11, 829-838.

Received 15 July 1996; revised 28 October 1996; accepted 5 November 1996. 